\title{
Testing hypotheses in the Birnbaum-Saunders distribution under type-II censored samples
}

\author{
Artur J. Lemonte, Silvia L. P. Ferrari \\ Departamento de Estatística, Universidade de São Paulo, Brazil
}

\begin{abstract}
The two-parameter Birnbaum-Saunders distribution has been used succesfully to model fatigue failure times. Although censoring is typical in reliability and survival studies, little work has been published on the analysis of censored data for this distribution. In this paper, we address the issue of performing testing inference on the two parameters of the Birnbaum-Saunders distribution under type-II right censored samples. The likelihood ratio statistic and a recently proposed statistic, the gradient statistic, provide a convenient framework for statistical inference in such a case, since they do not require to obtain, estimate or invert an information matrix, which is an advantage in problems involving censored data. An extensive Monte Carlo simulation study is carried out in order to investigate and compare the finite sample performance of the likelihood ratio and the gradient tests. Our numerical results show evidence that the gradient test should be preferred. Three empirical applications are presented.
\end{abstract}

Key words: Birnbaum-Saunders distribution; Censored data; Fatigue life distribution; Lifetime data; Likelihood ratio test; Maximum likelihood estimation; Monte Carlo simulations; Gradient test.

\section{Introduction}

Birnbaum and Saunders (1969a, b) proposed a family of two-parameter distributions to model failure time due to fatigue under cyclic loading and the assumption that failure follows from the development and growth of a dominant crack. This distribution is known as the two-parameter Birnbaum-Saunders $(\mathcal{B S})$ distribution or as the fatigue life distribution. The $\mathcal{B S}$ distribution is an attractive alternative to the Weibull, gamma, and log-normal models, since its derivation considers the basic characteristics of the fatigue process. A more general derivation was provided by Desmond (1985) based on a biological model and relaxing several of the assumptions made by Birnbaum and Saunders (1969a). Desmond (1986) investigated the relationship between the $\mathcal{B S}$ distribution and the inverse Gaussian distribu- 
tion and demonstrated that the $\mathcal{B S}$ distribution is an equal-weight mixture of an inverse Gaussian distribution and its complementary reciprocal; see also Bhattacharyya and Fries (1982).

The random variable $T$ is said to have a $\mathcal{B S}$ distribution with parameters $\alpha, \beta>0$, say $\mathcal{B S}(\alpha, \beta)$, if its cumulative distribution function (cdf) is given by

$$
F(t)=\Phi(v), \quad t>0
$$

where $\Phi(\cdot)$ is the standard normal distribution function, $v=\rho(t / \beta) / \alpha, \rho(z)=z^{1 / 2}-z^{-1 / 2}$ and $\alpha$ and $\beta$ are shape and scale parameters, respectively. Also, $\beta$ is the median of the distribution: $F(\beta)=\Phi(0)=1 / 2$. For any constant $k>0$, it follows that $k T \sim \mathcal{B S}(\alpha, k \eta)$. It is noteworthy that the reciprocal property holds for the $\mathcal{B S}$ distribution: $T^{-1} \sim \mathcal{B S}\left(\alpha, \beta^{-1}\right)$; see Saunders (1974). The probability density and hazard ratio functions corresponding to (1) are given by

$$
f(t)=\kappa(\alpha, \beta) t^{-3 / 2}(t+\beta) \exp \left\{-\frac{\tau(t / \beta)}{2 \alpha^{2}}\right\}, \quad t>0,
$$

and

$$
r(t)=\frac{\kappa(\alpha, \beta) t^{-3 / 2}(t+\beta) \exp \left\{-\tau(t / \beta) /\left(2 \alpha^{2}\right)\right\}}{1-\Phi(v)}, \quad t>0
$$

respectively, where $\kappa(\alpha, \beta)=\exp \left(\alpha^{-2}\right) /(2 \alpha \sqrt{2 \pi \beta})$ and $\tau(z)=z+z^{-1}$. The expected value, variance, skewness and kurtosis are, respectively, $\mathrm{E}(T)=\beta\left(1+\alpha^{2} / 2\right)$, $\operatorname{var}(T)=(\alpha \beta)^{2}\left(1+5 \alpha^{2} / 4\right)$, $\mu_{3}=16 \alpha^{2}\left(11 \alpha^{2}+6\right) /\left(5 \alpha^{2}+4\right)^{3}$ and $\mu_{4}=3+6 \alpha^{2}\left(93 \alpha^{2}+41\right) /\left(5 \alpha^{2}+4\right)^{2}$. A general expression to obtain the moments of (2) was derived by Rieck (1999) and is given by

$$
\mathrm{E}\left(T^{p}\right)=\beta^{p}\left(\frac{K_{p+1 / 2}\left(\alpha^{-2}\right)+K_{p-1 / 2}\left(\alpha^{-2}\right)}{2 K_{1 / 2}\left(\alpha^{-2}\right)}\right),
$$

where $K_{\nu}(\cdot)$ denotes the modified Bessel function of the third kind and order $\nu$. As noted before, if $T \sim \mathcal{B S}(\alpha, \beta)$, then $T^{-1} \sim \mathcal{B S}\left(\alpha, \beta^{-1}\right)$. It then follows that $\mathrm{E}\left(T^{-1}\right)=\beta^{-1}\left(1+\alpha^{2} / 2\right)$ and $\operatorname{var}\left(T^{-1}\right)=\alpha^{2} \beta^{-2}\left(1+5 \alpha^{2} / 4\right)$. The $\mathcal{B S}$ distribution is positively skewed and the asymmetry of the distribution decreases with $\alpha$. Figure 1 plots the density and hazard ratio function for some values of $\alpha$ with $\beta=1$. For applications of the $\mathcal{B S}$ distribution in reliability studies see, for example, Balakrishnan et al. (2007), and for its use outside this field see Leiva et al. (2008) and Leiva et al. (2009).

The $\mathcal{B S}$ distribution has received significant attention over the last few years. Kundu et al. (2008) discussed the shape of the hazard function of the $\mathcal{B S}$ distribution. Improved frequentist inference for the $\mathcal{B S}$ distribution is discussed in Wu and Wong (2004), Cysneiros et al. (2008) and Lemonte et al. (2007, 2008), while estimation using reference prior is presented in Xu and Tang (2010). Extensions of the $\mathcal{B S}$ distribution are proposed in Díaz-García and Leiva (2005), Gómes et al. (2009), Guiraud et al. (2009) and Castillo et al. (2009). Also, Bhatti (2010) introduced the $\mathcal{B S}$ autoregressive conditional duration model and Aslam et al. (2010) developed various acceptance sampling schemes based on the truncated life tests. 

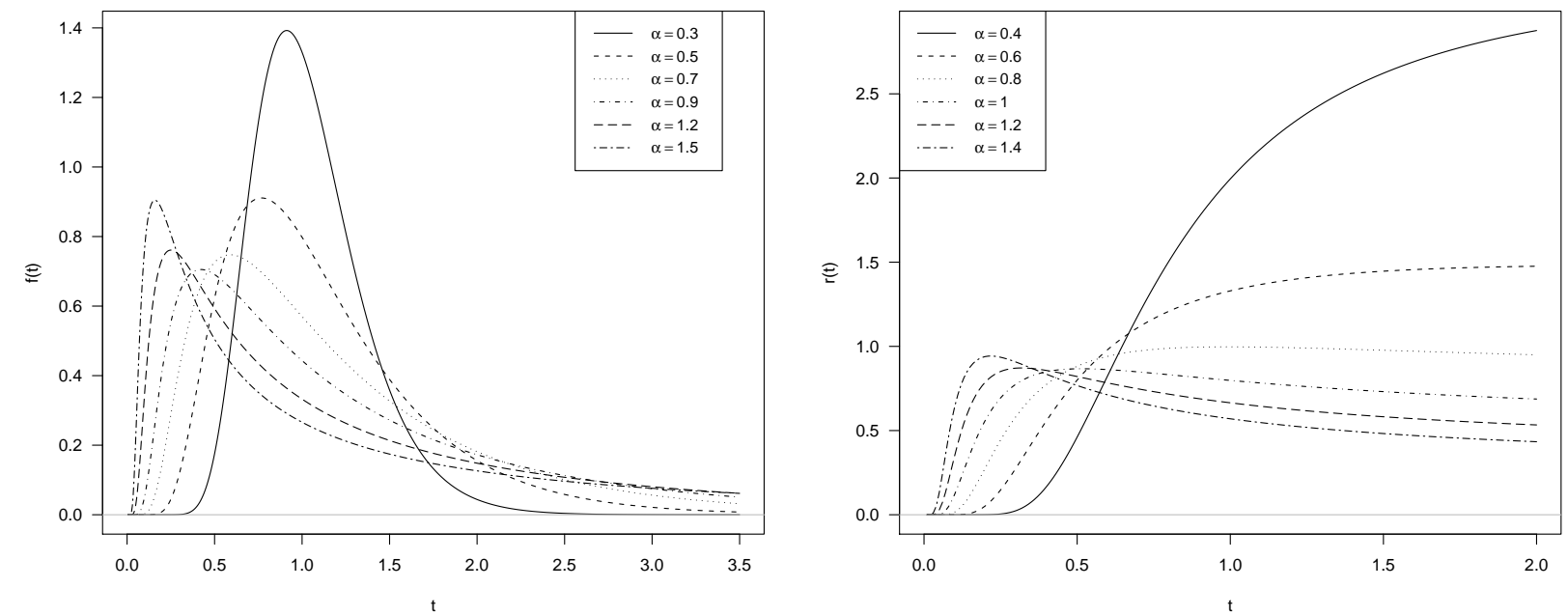

Figure 1: Plots of the density and hazard ratio function; $\beta=1$.

Little work has been published on the analysis of censored data for the $\mathcal{B S}$ distribution although censoring is common in reliability and survival studies. Rieck (1995) considered parametric estimation for the $\mathcal{B S}$ distribution based on type-II censored samples. Jeng (2003) explored and compared different procedures to compute confidence intervals for the parameters and quantiles of the $\mathcal{B S}$ distribution for complete and type-I censored data. Ng et al. (2006) and Wang et al. (2006) discussed the maximum likelihood estimation of the parameters of the $\mathcal{B S}$ distribution and proposed a simple bias-reduction method to reduce the bias of the maximum likelihood estimators (MLEs) based on type-II censored samples and random censoring, respectively. Also, From and Li (2006) discussed the estimation of the parameters of the $\mathcal{B S}$ distribution under censored samples.

A particularly useful censoring mechanism is the type-II (right) censoring, which occurs when $n$ items are placed on test and the experiment is terminated when the first $m(m<n)$ items fail. Since life testing is often time consuming and expensive, type-II censoring may be used to reduce testing time and costs. This paper focus on testing inference for the $\mathcal{B S}$ distribution under type-II censored samples.

A commonly used test procedure is the likelihood ratio test. A new criterion for testing hypotheses, referred to as the gradient test, was proposed by Terrell (2002). Its statistic shares the same first order asymptotic properties with the likelihood ratio statistic. Like the likelihood ratio statistic and unlike the score and the Wald statistics, the gradient statistic does not require computation of the information matrix, neither observed nor expected, which is an advantage in problems involving censored samples. The chief goal of our paper is to present Monte Carlo simulations in order to evaluate and compare the performance of the likelihood ratio and gradient tests for the $\mathcal{B S}$ distribution under type-II right censored samples. The other two tests will be briefly taken into account in our simulation study. To the best of our knowledge, there is no mention in the statistical literature on the use of the 
gradient test under censoring.

The paper is organized as follows. Section 2 briefly describes the likelihood ratio and gradient tests. Section 3 presents inference on the parameters of the $\mathcal{B S}$ distribution under type-II right censored samples. Numerical results are presented and discussed in Section 4. We evaluate and compare the performance of the likelihood ratio and gradient tests for testing hypotheses on the parameters of the $\mathcal{B S}$ distribution under type-II right censored samples. Applications are presented in Section 5 , Finally, Section 6 closes the paper with some conclusions.

\section{Likelihood ratio and gradient tests}

Let $\ell(\boldsymbol{\theta})$ denote the total $\log$-likelihood function and consider the partition $\boldsymbol{\theta}=\left(\boldsymbol{\theta}_{1}^{\top}, \boldsymbol{\theta}_{2}^{\top}\right)^{\top}$, where the dimensions of $\boldsymbol{\theta}_{1}$ and $\boldsymbol{\theta}_{2}$ are $q$ and $p-q$, respectively, i.e. $\boldsymbol{\theta}$ is a $p$-vector of unknown parameters. Let $\boldsymbol{U}_{\boldsymbol{\theta}}$ denote the score function for $\boldsymbol{\theta}$. The partition for $\boldsymbol{\theta}$ induces the corresponding partition: $\boldsymbol{U}_{\boldsymbol{\theta}}=\left(\boldsymbol{U}_{\boldsymbol{\theta}_{1}}^{\top}, \boldsymbol{U}_{\boldsymbol{\theta}_{2}}^{\top}\right)^{\top}$. Suppose the interest lies in testing the composite null hypothesis

$$
\mathcal{H}_{0}: \boldsymbol{\theta}_{2}=\boldsymbol{\theta}_{20}
$$

against $\mathcal{H}_{1}: \boldsymbol{\theta}_{2} \neq \boldsymbol{\theta}_{20}$, where $\boldsymbol{\theta}_{20}$ is a specified vector. Hence, $\boldsymbol{\theta}_{1}$ is a vector of nuisance parameters. The likelihood ratio $(L R)$ and gradient $\left(S_{T}\right)$ statistics for testing $\mathcal{H}_{0}$ versus $\mathcal{H}_{1}$ are given, respectively, by

$$
L R=2\{\ell(\widehat{\boldsymbol{\theta}})-\ell(\widetilde{\boldsymbol{\theta}})\}, \quad S_{T}=\widetilde{\boldsymbol{U}}_{\boldsymbol{\theta}}^{\top}(\widehat{\boldsymbol{\theta}}-\widetilde{\boldsymbol{\theta}}),
$$

where $\widehat{\boldsymbol{\theta}}=\left(\widehat{\boldsymbol{\theta}}_{1}^{\top}, \widehat{\boldsymbol{\theta}}_{2}^{\top}\right)^{\top}$ and $\widetilde{\boldsymbol{\theta}}=\left(\widetilde{\boldsymbol{\theta}}_{1}^{\top}, \boldsymbol{\theta}_{20}^{\top}\right)^{\top}$ denote the MLEs of $\boldsymbol{\theta}=\left(\boldsymbol{\theta}_{1}^{\top}, \boldsymbol{\theta}_{2}^{\top}\right)^{\top}$ under $\mathcal{H}_{1}$ and $\mathcal{H}_{0}$, respectively, and $\widetilde{\boldsymbol{U}}_{\boldsymbol{\theta}}=\boldsymbol{U}_{\boldsymbol{\theta}}(\widetilde{\boldsymbol{\theta}})=\left(\widetilde{\boldsymbol{U}}_{\boldsymbol{\theta}_{1}}^{\top}, \widetilde{\boldsymbol{U}}_{\boldsymbol{\theta}_{2}}^{\top}\right)^{\top}$, with $\widetilde{\boldsymbol{U}}_{\boldsymbol{\theta}_{1}}=\boldsymbol{U}_{\boldsymbol{\theta}_{1}}(\widetilde{\boldsymbol{\theta}})$ and $\widetilde{\boldsymbol{U}}_{\boldsymbol{\theta}_{2}}=\boldsymbol{U}_{\boldsymbol{\theta}_{2}}(\widetilde{\boldsymbol{\theta}})$. Since $\widetilde{\boldsymbol{U}}_{\boldsymbol{\theta}_{1}}=\mathbf{0}$, the gradient statistic can be written as $S_{T}=\widetilde{\boldsymbol{U}}_{\boldsymbol{\theta}_{2}}^{\top}\left(\widehat{\boldsymbol{\theta}}_{2}-\boldsymbol{\theta}_{20}\right)$. The limiting distribution of $L R$ and $S_{T}$ is $\chi_{p-q}^{2}$ under $\mathcal{H}_{0}$ and $\chi_{p-q, \lambda}^{2}$, i.e. a noncentral chi-square distribution with $p-q$ degrees of freedom and an appropriate noncentrality parameter $\lambda$, under $\mathcal{H}_{1}$. The null hypothesis is rejected for a given nominal level, $\gamma$ say, if the test statistic exceeds the upper $100(1-\gamma) \%$ quantile of the $\chi_{p-q}^{2}$ distribution.

Terrell (2002) points out that the gradient statistic "is not transparently non-negative, even though it must be so asymptotically." His Theorem 2 implies that if the log-likelihood function is concave and is differentiable at $\widetilde{\boldsymbol{\theta}}$, then $S_{T} \geq 0$. Recently, Lemonte and Ferrari (2010a) derived the nonnull asymptotic expansion of the distribution of the gradient test statistic for a composite hypothesis under a sequence of local alternative hypotheses converging to the null hypothesis at rate $n^{-1 / 2}$. The authors also compared the local powers of the gradient, likelihood ratio, Wald and score tests. In general, no test is uniformly better than the others as far as local power is concerned. Lemonte and Ferrari (2010b) obtained the nonnull asymptotic expansions of the likelihood ratio, Wald, score and gradient statistics in Birnbaum-Saunders regression models when no censoring is present. An interesting finding is that, up to an error of order $n^{-1}$, the four tests have the same local power in this class of models. Their 
simulation study evidenced that the score and the gradient tests perform better than the likelihood ratio and Wald tests in small and moderate-sized samples.

\section{Inference under type-II censored samples}

Let $\left\{t_{1}, \ldots, t_{m}\right\}$ be an ordered type-II right censored random sample obtained from $n$ units placed on a life-testing experiment wherein each unit has its lifetime following the $\mathcal{B S}$ distribution, with the largest $(n-m)$ lifetimes having been censored. Let $\boldsymbol{\theta}=(\alpha, \beta)^{\top}$ be the parameter vector. For notational convenience, let

$$
H(z)=\frac{\phi(z)}{1-\Phi(z)}, \quad h(z)=\frac{\tau\left(z^{1 / 2}\right)}{\alpha}, \quad t_{i}^{*}=\frac{t_{i}}{\beta},
$$

where $\phi(\cdot)$ is the standard normal density function.

The likelihood function can be written as (Balakrishnan and Cohen, 1991)

$$
L(\boldsymbol{\theta})=\left\{\prod_{i=1}^{m} \kappa(\alpha, \beta) t_{i}^{-3 / 2}\left(t_{i}+\beta\right) \exp \left(-\frac{\tau\left(t_{i}^{*}\right)}{2 \alpha^{2}}\right)\right\} \frac{n !\left\{1-\Phi\left(v_{m}\right)\right\}^{n-m}}{m !(n-m) !}
$$

where $v_{m}=\rho\left(t_{m}^{*}\right) / \alpha$. Thus, the log-likelihood function, except for a constant term, is given by

$$
\begin{aligned}
\ell(\boldsymbol{\theta})=\ell(\alpha, \beta)= & m \log \{\kappa(\alpha, \beta)\}+\sum_{i=1}^{m} \log \left(t_{i}+\beta\right)-\frac{1}{2 \alpha^{2}} \sum_{i=1}^{m} \tau\left(t_{i}^{*}\right) \\
& +(n-m) \log \left\{1-\Phi\left(v_{m}\right)\right\} .
\end{aligned}
$$

By taking partial derivatives of the log-likelihood function with respect to $\alpha$ and $\beta$ we obtain the components of the score vector, $\boldsymbol{U}_{\boldsymbol{\theta}}=\left(U_{\alpha}, U_{\beta}\right)^{\top}$ :

$$
\begin{gathered}
U_{\alpha}=-\frac{m}{\alpha}\left(1+\frac{2}{\alpha^{2}}\right)+\frac{1}{\alpha^{3}} \sum_{i=1}^{m} \tau\left(t_{i}^{*}\right)+\frac{(n-m) v_{m} H\left(v_{m}\right)}{\alpha} \\
U_{\beta}=-\frac{m}{2 \beta}+\sum_{i=1}^{m} \frac{1}{t_{i}+\beta}+\frac{1}{2 \alpha^{2} \beta} \sum_{i=1}^{m} \rho\left(t_{i}^{* 2}\right)+\frac{(n-m) h\left(t_{m}^{*}\right) H\left(v_{m}\right)}{2 \beta} .
\end{gathered}
$$

Setting $U_{\alpha}$ and $U_{\beta}$ equal to zero yields the MLE $\widehat{\boldsymbol{\theta}}=(\widehat{\alpha}, \widehat{\beta})^{\top}$ of $\boldsymbol{\theta}=(\alpha, \beta)^{\top}$. These equations cannot be solved analytically and statistical software can be used to solve them numerically via iterative methods. The BFGS method (see, for example, Nocedal and Wright, 1999; Press et al., 2007) with analytical derivatives has been used for maximizing the log-likelihood function $\ell(\boldsymbol{\theta})$.

We now consider hypothesis testing on the parameters $\alpha$ and $\beta$ based on the likelihood ratio and gradient statistics. The interest lies in testing the null hypotheses

$$
\mathcal{H}_{00}: \alpha=\alpha^{(0)}, \quad \mathcal{H}_{01}: \beta=\beta^{(0)},
$$


which are tested against $\mathcal{H}_{10}: \alpha \neq \alpha^{(0)}$ and $\mathcal{H}_{11}: \beta \neq \beta^{(0)}$, respectively. Here $\alpha^{(0)}$ and $\beta^{(0)}$ are positive known scalars. For testing $\mathcal{H}_{00}$, the likelihood ratio and gradient statistics are given by

$$
L R_{(\alpha)}=2\left\{\ell(\widehat{\beta}, \widehat{\alpha})-\ell\left(\alpha^{(0)}, \widetilde{\beta}\right)\right\}, \quad S_{T(\alpha)}=\widetilde{U}_{\alpha}\left(\widehat{\alpha}-\alpha^{(0)}\right),
$$

where $\widehat{\alpha}$ and $\widehat{\beta}$ are the unrestricted MLEs obtained from the maximization of $\ell(\boldsymbol{\theta})$ under the alternative hypotheses, $\widetilde{\beta}$ is the restricted MLE obtained from the maximization of $\ell(\boldsymbol{\theta})$ under $\mathcal{H}_{00}$ and $\widetilde{U}_{\alpha}=$ $U_{\alpha}\left(\alpha^{(0)}, \widetilde{\beta}\right)$. For testing $\mathcal{H}_{01}$, we have

$$
L R_{(\beta)}=2\left\{\ell(\widehat{\beta}, \widehat{\alpha})-\ell\left(\widetilde{\alpha}, \beta^{(0)}\right)\right\}, \quad S_{T(\beta)}=\widetilde{U}_{\beta}\left(\widehat{\beta}-\beta^{(0)}\right) .
$$

Here, $\widetilde{\alpha}$ is the restricted MLE obtained from the maximization of $\ell(\boldsymbol{\theta})$ under the hypothesis $\mathcal{H}_{01}$ and $\widetilde{U}_{\beta}=U_{\beta}\left(\widetilde{\alpha}, \beta^{(0)}\right)$. The limiting distribution of these statistics is $\chi_{1}^{2}$ under the respective null hypothesis. In both cases, the null hypothesis is rejected if the chosen test statistic exceeds the upper $100(1-\gamma) \%$ quantile of the $\chi_{1}^{2}$ distribution.

Theorem 3 in Terrell (2002) points out an important feature of the gradient test. It suggests that we can, in general, improve the approximation of the distribution of the gradient statistic by a chi-square distribution under the null hypothesis by using a less biased estimator. On the other hand, Theorem 2 in Terrell (2002) indicates that if the estimate used in the gradient statistic is not the maximum likelihood, the non-negativity of the gradient statistic is not guaranteed.

A bias-corrected estimator for $\alpha$ was derived in $\mathrm{Ng}$ et al. (2006) and is given by

$$
\bar{\alpha}=\widehat{\alpha}\left\{1-\frac{1}{n}\left[1+2.5\left(1-\frac{m}{n}\right)\right]\right\}^{-1} .
$$

The authors showed through Monte Carlo simulations that $\bar{\alpha}$ is less biased than the original MLE, $\widehat{\alpha}$, of $\alpha$. Therefore, based on this estimator, we can define the adjusted gradient statistic $S_{T(\alpha)}^{*}=$ $\widetilde{U}_{\alpha}\left(\bar{\alpha}-\alpha^{(0)}\right)$ for testing the null hypothesis $\mathcal{H}_{00}: \alpha=\alpha^{(0)}$. The limiting distribution of $S_{T(\alpha)}^{*}$ is also $\chi_{1}^{2}$ under $\mathcal{H}_{00}$. It is expected that the gradient test that uses the statistic $S_{T(\alpha)}^{*}$ have better size performance than the gradient test based on the original gradient statistic $S_{T(\alpha)}$. A disadvantage of the adjusted gradient statistic $S_{T(\alpha)}^{*}$ is that it can take on negative values. However, in order to avoid negative values we shall redefine $S_{T(\alpha)}^{*}$ as

$$
S_{T(\alpha)}^{*}=\max \left\{0, \widetilde{U}_{\alpha}\left(\bar{\alpha}-\alpha^{(0)}\right)\right\} .
$$

An adjusted gradient statistic for testing the null hypothesis $\mathcal{H}_{01}: \beta=\beta^{(0)}$ is not considered here because a bias-corrected estimator for $\beta$ is not available. In the next section, we shall present an extensive Monte Carlo study in order to evaluate and compare the performance of the tests presented in this section.

\section{Numerical results}

In this section we shall present an extensive Monte Carlo simulation study in which we evaluate and compare the finite sample performance of the likelihood ratio and gradient tests for testing hypothe- 
ses on the parameters of the $\mathcal{B S}$ distribution under type-II right censored samples. We set the degree of censoring (d.o.c.) at $0(10) 50 \%$, the sample size at $n=20$ and 40, and the shape parameter at $\alpha=0.1,0.3,0.5,0.75$ and 1.0. Without loss of generality, the scale parameter $\beta$ was kept fixed at 1.0. The nominal levels of the tests were $\gamma=10 \%$ and 5\%. The number of Monte Carlo replications was 10000. All the Monte Carlo simulation experiments were performed using the Ox matrix programming language (Doornik, 2006). Ox is freely distributed for academic purposes and available at http://www.doornik.com.

Table 1 presents the null rejection rates (entries are percentages) of the tests which are based on the statistics $L R_{(\alpha)}, S_{T(\alpha)}$ and $S_{T(\alpha)}^{*}$ for testing the null hypothesis $\mathcal{H}_{00}: \alpha=\alpha^{(0)}$. Our main findings are as follows. First, for complete data without censoring (d.o.c. $=0 \%$ ), the tests based on $S_{T(\alpha)}$ and $S_{T(\alpha)}^{*}$ are less size distorted than the test that uses $L R_{(\alpha)}$; in fact, they produce null rejection rates that are very close to the nominal levels in all the cases considered. In addition, the adjusted gradient test is slightly superior than the original gradient test. For example, for $n=20, \alpha^{(0)}=0.5$ and $\gamma=10 \%$, the null rejection rates are $11.48 \%\left(L R_{(\alpha)}\right), 9.65 \%\left(S_{T(\alpha)}\right)$ and $9.81 \%\left(S_{T(\alpha)}^{*}\right)$. Second, the size distortion of all tests increases with the d.o.c., the likelihood ratio test and the original gradient test displaying null rejection rates that are, respectively, greater and smaller than the nominal level. The adjusted gradient test is the less size distorted in most of the cases. For instance, for $n=20$, $\alpha^{(0)}=0.75$ and $\gamma=5 \%$, the null rejection rates are $6.17 \%\left(L R_{(\alpha)}\right), 4.28 \%\left(S_{T(\alpha)}\right)$ and $4.77 \%\left(S_{T(\alpha)}^{*}\right)$ for d.o.c. $=10 \%$, and $7.22 \%\left(L R_{(\alpha)}\right), 4.15 \%\left(S_{T(\alpha)}\right)$ and $4.96 \%\left(S_{T(\alpha)}^{*}\right)$ for d.o.c. $=30 \%$. Note that all the tests become less size distorted as the sample size increases, as expected.

The null rejection rates of the tests which are based on $L R_{(\beta)}$ and $S_{T(\beta)}$ for testing the null hypothesis $\mathcal{H}_{01}: \beta=1$ are presented in Table 2. For complete data without censoring (d.o.c. $=0 \%$ ), the gradient test is less size distorted than the likelihood test in all the cases. For example, for $n=20$, $\alpha=0.5$ and $\gamma=10 \%$, the null rejection rates are $11.98 \%\left(L R_{(\beta)}\right)$ and $10.53 \%\left(S_{T(\beta)}\right)$. Additionally, the size distortion of the tests increases with the degree of censoring and, similarly to what occurs for testing on the parameter $\alpha$, the likelihood ratio test presented a liberal behavior, while the gradient test was conservative in the majority of the cases. For instance, when $n=20, \alpha=0.5$ and $\gamma=10 \%$, the null rejection rates are $12.28 \%\left(L R_{(\beta)}\right)$ and $9.88 \%\left(S_{T(\beta)}\right)$ for d.o.c. $=20 \%$ and $13.57 \%\left(L R_{(\beta)}\right)$ and $9.47 \%\left(S_{T(\beta)}\right)$ for d.o.c. $=50 \%$. It should be noticed that the gradient test is less size distorted than the likelihood test in all the cases considered. Again, the tests become less size distorted as the sample size increases.

Table 3 contains the nonnull rejection rates (powers) of the tests. We set $\alpha=0.5, \beta=1$, $n=80,120$ and 150, and the degree of censoring at $0 \%, 20 \%$ and $40 \%$. The rejection rates were obtained under the alternative hypotheses $\mathcal{H}_{10}: \alpha=\delta_{1}$ and $\mathcal{H}_{11}: \beta=\delta_{2}$, for different values of $\delta_{1}$ and $\delta_{2}$. The test that uses $L R_{(\alpha)}$ presented smaller powers for testing hypotheses on the parameter $\alpha$ than the tests which are based on the statistics $S_{T(\alpha)}$ and $S_{T(\alpha)}^{*}$. For example, when $n=80$, d.o.c. $=$ $20 \%$ and $\delta_{1}=0.60$, the nonnull rejection rates are $58.92 \%\left(L R_{(\alpha)}\right), 62.02 \%\left(S_{T(\alpha)}\right)$ and $65.53 \%$ $\left(S_{T(\alpha)}^{*}\right)$. Additionally, the tests which use $L R_{(\beta)}$ and $S_{T(\beta)}$ for testing hypothesis on the parameter 
Table 1: Null rejection rates $(\%)$ for $\mathcal{H}_{00}: \alpha=\alpha^{(0)}$.

\begin{tabular}{|c|c|c|c|c|c|c|c|c|c|c|c|c|c|}
\hline \multirow{3}{*}{$\begin{array}{c}\text { d.o.c. } \\
(\%)\end{array}$} & \multirow[b]{3}{*}{$\alpha^{(0)}$} & \multicolumn{6}{|c|}{$n=20$} & \multicolumn{6}{|c|}{$n=40$} \\
\hline & & \multicolumn{2}{|c|}{$L R_{(\alpha)}$} & \multicolumn{2}{|c|}{$S_{T(\alpha)}$} & \multicolumn{2}{|c|}{$S_{T(\alpha)}^{*}$} & \multicolumn{2}{|c|}{$L R_{(\alpha)}$} & \multicolumn{2}{|c|}{$S_{T(\alpha)}$} & \multicolumn{2}{|c|}{$S_{T(\alpha)}^{*}$} \\
\hline & & $10 \%$ & $5 \%$ & $10 \%$ & $5 \%$ & $10 \%$ & $5 \%$ & $10 \%$ & $5 \%$ & $10 \%$ & $5 \%$ & $10 \%$ & $5 \%$ \\
\hline \multirow[t]{5}{*}{0} & 0.1 & 11.53 & 6.07 & 9.70 & 4.25 & 9.83 & 4.52 & 10.76 & 5.23 & 9.67 & 4.82 & 9.81 & 5.16 \\
\hline & 0.3 & 11.50 & 6.10 & 9.70 & 4.22 & 9.78 & 4.48 & 10.74 & 5.22 & 9.69 & 4.83 & 9.83 & 5.15 \\
\hline & 0.5 & 11.48 & 6.15 & 9.65 & 4.21 & 9.81 & 4.45 & 10.75 & 5.24 & 9.71 & 4.80 & 9.85 & 5.12 \\
\hline & 0.75 & 11.55 & 6.24 & 9.58 & 4.22 & 9.78 & 4.41 & 10.77 & 5.29 & 9.74 & 4.79 & 9.83 & 5.09 \\
\hline & 1.0 & 11.73 & 6.25 & 9.62 & 4.26 & 9.78 & 4.46 & 10.84 & 5.36 & 9.67 & 4.81 & 9.79 & 5.10 \\
\hline \multirow[t]{5}{*}{10} & 0.1 & 11.85 & 6.12 & 9.57 & 4.29 & 9.84 & 4.90 & 10.79 & 5.70 & 9.63 & 4.61 & 9.87 & 4.79 \\
\hline & 0.3 & 11.86 & 6.12 & 9.55 & 4.29 & 9.82 & 4.88 & 10.77 & 5.70 & 9.62 & 4.60 & 9.85 & 4.75 \\
\hline & 0.5 & 11.85 & 6.15 & 9.59 & 4.29 & 9.81 & 4.85 & 10.78 & 5.67 & 9.63 & 4.56 & 9.80 & 4.73 \\
\hline & 0.75 & 11.96 & 6.17 & 9.59 & 4.28 & 9.82 & 4.77 & 10.76 & 5.66 & 9.59 & 4.51 & 9.88 & 4.74 \\
\hline & 1.0 & 12.09 & 6.19 & 9.58 & 4.35 & 9.77 & 4.73 & 10.90 & 5.69 & 9.67 & 4.51 & 9.89 & 4.73 \\
\hline \multirow[t]{5}{*}{20} & 0.1 & 12.26 & 6.50 & 9.80 & 4.10 & 10.01 & 5.09 & 10.71 & 5.30 & 9.23 & 4.36 & 9.38 & 4.63 \\
\hline & 0.3 & 12.24 & & 9.74 & 4.12 & 9.96 & & 10.77 & 5.26 & 9.24 & 4.36 & 9.29 & 4.63 \\
\hline & 0.5 & 12.27 & 6.50 & 9.79 & 4.16 & 9.94 & 5.04 & 10.78 & 5.25 & 9.29 & 4.37 & 9.19 & 4.63 \\
\hline & 0.75 & 12.26 & 6.55 & 9.68 & 4.20 & 9.91 & 5.00 & 10.82 & 5.36 & 9.32 & 4.27 & 9.33 & 4.55 \\
\hline & 1.0 & 12.27 & 6.64 & 9.72 & 4.22 & 9.81 & 4.93 & 10.85 & 5.36 & 9.26 & 4.28 & 9.21 & 4.52 \\
\hline \multirow[t]{5}{*}{30} & 0.1 & 12.98 & 6.98 & 9.76 & 4.30 & 10.30 & 5.12 & 11.10 & 5.55 & 9.40 & 4.43 & 9.72 & 4.64 \\
\hline & 0.3 & 13.04 & 6.96 & 9.74 & 4.26 & 10.17 & 5.10 & 11.11 & 5.52 & 9.40 & 4.33 & 9.69 & 4.67 \\
\hline & 0.5 & 13.04 & 7.06 & 9.82 & 4.28 & 10.14 & 5.04 & 10.98 & 5.50 & 9.38 & 4.30 & 9.66 & 4.73 \\
\hline & 0.75 & 13.04 & 7.22 & 9.81 & 4.15 & 9.96 & 4.96 & 10.97 & 5.58 & 9.34 & 4.24 & 9.56 & 4.63 \\
\hline & 1.0 & 13.08 & 7.26 & 9.76 & 4.19 & 9.76 & 4.91 & 10.90 & 5.59 & 9.24 & 4.24 & 9.27 & 4.60 \\
\hline \multirow[t]{5}{*}{40} & 0.1 & 13.30 & 7.23 & 9.42 & 4.27 & 9.93 & 5.15 & 11.78 & 5.89 & 9.96 & 4.79 & 9.93 & 5.38 \\
\hline & 0.3 & 13.31 & 7.25 & 9.40 & 4.21 & 9.95 & 5.18 & 11.75 & 5.89 & 9.98 & 4.81 & 9.95 & 5.36 \\
\hline & 0.5 & 13.29 & 7.12 & 9.42 & 4.14 & 9.92 & 5.13 & 11.74 & 5.93 & 10.00 & 4.75 & 9.88 & 5.26 \\
\hline & 0.75 & 13.25 & 7.26 & 9.32 & 4.05 & 9.90 & 5.04 & 11.73 & 5.87 & 9.83 & 4.78 & 9.82 & 5.24 \\
\hline & 1.0 & 13.52 & 7.32 & 9.20 & 3.95 & 9.73 & 4.95 & 11.73 & 5.95 & 9.73 & 4.68 & 9.74 & 5.09 \\
\hline \multirow[t]{5}{*}{50} & 0.1 & 14.35 & 7.99 & 9.30 & 3.95 & 9.78 & 4.91 & 11.42 & 6.02 & 9.38 & 4.57 & 9.61 & 5.10 \\
\hline & 0.3 & 14.37 & 7.94 & 9.24 & 3.88 & 9.70 & 4.84 & 11.39 & 6.05 & 9.42 & 4.61 & 9.64 & 5.07 \\
\hline & 0.5 & 14.18 & 7.92 & 9.20 & 3.89 & 9.65 & 4.85 & 11.39 & 6.12 & 9.33 & 4.55 & 9.57 & 5.03 \\
\hline & 0.75 & 14.37 & 8.01 & 9.04 & 3.81 & 9.56 & 4.71 & 11.42 & 6.17 & 9.39 & 4.45 & 9.39 & 5.02 \\
\hline & 1.0 & 14.60 & 8.05 & 8.67 & 3.58 & 9.23 & 4.59 & 11.51 & 6.23 & 9.12 & 4.33 & 9.22 & 4.91 \\
\hline
\end{tabular}


Table 2: Null rejection rates $(\%)$ for $\mathcal{H}_{01}: \beta=1$.

\begin{tabular}{|c|c|c|c|c|c|c|c|c|c|}
\hline \multirow{3}{*}{$\begin{array}{c}\text { d.o.c. } \\
(\%)\end{array}$} & \multirow[b]{3}{*}{$\alpha$} & \multicolumn{4}{|c|}{$n=20$} & \multicolumn{4}{|c|}{$n=40$} \\
\hline & & \multicolumn{2}{|c|}{$L R_{(\beta)}$} & \multicolumn{2}{|c|}{$S_{T(\beta)}$} & \multicolumn{2}{|c|}{$L R_{(\beta)}$} & \multicolumn{2}{|c|}{$S_{T(\beta)}$} \\
\hline & & $10 \%$ & $5 \%$ & $10 \%$ & $5 \%$ & $10 \%$ & $5 \%$ & $10 \%$ & $5 \%$ \\
\hline \multirow[t]{5}{*}{0} & 0.1 & 12.06 & 6.43 & 10.74 & 4.98 & 11.00 & 5.43 & 10.38 & 4.92 \\
\hline & 0.3 & 11.95 & 6.44 & 10.57 & 5.03 & 11.02 & 5.44 & 10.37 & 4.94 \\
\hline & 0.5 & 11.98 & 6.52 & 10.53 & 4.94 & 11.00 & 5.50 & 10.40 & 4.95 \\
\hline & 0.75 & 11.78 & 6.49 & 10.33 & 4.89 & 11.14 & 5.56 & 10.26 & 5.08 \\
\hline & 1.0 & 11.74 & 6.74 & 10.21 & 4.93 & 11.23 & 5.81 & 10.33 & 5.08 \\
\hline \multirow[t]{5}{*}{10} & 0.1 & 12.00 & 6.44 & 10.69 & 4.70 & 10.82 & 5.59 & 10.17 & 4.76 \\
\hline & 0.3 & 12.01 & 6.40 & 10.40 & 4.70 & 10.80 & 5.51 & 10.16 & 4.82 \\
\hline & 0.5 & 11.96 & 6.40 & 10.22 & 4.63 & 10.82 & 5.45 & 10.18 & 4.81 \\
\hline & 0.75 & 11.91 & 6.27 & 9.89 & 4.51 & 11.02 & 5.51 & 10.15 & 4.83 \\
\hline & 1.0 & 11.87 & 6.29 & 9.78 & 4.60 & 11.09 & 5.64 & 10.05 & 4.80 \\
\hline \multirow[t]{5}{*}{20} & 0.1 & 12.24 & 6.44 & 10.08 & 4.57 & 11.13 & 5.55 & 10.07 & 4.75 \\
\hline & 0.3 & 12.31 & 6.41 & 10.00 & 4.68 & 11.14 & 5.52 & 9.96 & 4.65 \\
\hline & 0.5 & 12.28 & 6.36 & 9.88 & 4.52 & 11.00 & 5.55 & 9.96 & 4.56 \\
\hline & 0.75 & 12.32 & 6.39 & 9.72 & 4.59 & 11.01 & 5.59 & 9.81 & 4.58 \\
\hline & 1.0 & 12.32 & 6.53 & 9.46 & 4.44 & 11.16 & 5.47 & 9.80 & 4.61 \\
\hline \multirow[t]{5}{*}{30} & 0.1 & 12.87 & 6.95 & 10.53 & 4.66 & 11.17 & 5.60 & 9.97 & 4.55 \\
\hline & 0.3 & 12.92 & 6.97 & 10.23 & 4.50 & 11.18 & 5.62 & 9.95 & 4.63 \\
\hline & 0.5 & 12.99 & 6.99 & 9.98 & 4.39 & 11.11 & 5.63 & 9.81 & 4.57 \\
\hline & 0.75 & 13.01 & 7.08 & 9.70 & 4.31 & 11.01 & 5.59 & 9.65 & 4.47 \\
\hline & 1.0 & 13.12 & 6.91 & 9.41 & 4.39 & 11.07 & 5.69 & 9.36 & 4.45 \\
\hline \multirow[t]{5}{*}{40} & 0.1 & 13.14 & 7.08 & 10.11 & 4.38 & 11.32 & 5.84 & 9.90 & 4.45 \\
\hline & 0.3 & 13.14 & 7.05 & 9.67 & 4.38 & 11.32 & 5.85 & 9.65 & 4.40 \\
\hline & 0.5 & 13.12 & 7.05 & 9.40 & 4.26 & 11.31 & 5.85 & 9.59 & 4.34 \\
\hline & 0.75 & 13.10 & 7.03 & 9.05 & 4.29 & 11.22 & 5.90 & 9.52 & 4.41 \\
\hline & 1.0 & 13.14 & 6.99 & 8.80 & 4.39 & 11.17 & 5.92 & 9.34 & 4.24 \\
\hline \multirow[t]{5}{*}{50} & 0.1 & 13.49 & 7.85 & 9.90 & 4.22 & 11.70 & 5.98 & 10.08 & 4.49 \\
\hline & 0.3 & 13.52 & 7.82 & 9.67 & 4.06 & 11.71 & 5.98 & 9.88 & 4.42 \\
\hline & 0.5 & 13.57 & 7.75 & 9.47 & 4.24 & 11.71 & 5.97 & 9.70 & 4.41 \\
\hline & 0.75 & 13.54 & 7.73 & 8.81 & 4.06 & 11.75 & 6.02 & 9.38 & 4.34 \\
\hline & 1.0 & 13.58 & 7.69 & 8.19 & 4.23 & 11.74 & 6.03 & 9.02 & 4.33 \\
\hline
\end{tabular}


$\beta$ have similar powers, the gradient test being slightly more powerful than the likelihood test. For instance, when $n=150$, d.o.c. $=0 \%$ and $\delta_{2}=1.13$, the nonnull rejection rates are $92.02 \%\left(L R_{(\beta)}\right)$ and $92.33 \%\left(S_{T(\beta)}\right)$. Note that the powers of the all the tests decrease as the degree of censoring increases. We also note that the powers of the tests increase with $n$ and also with $\delta_{1}$ and $\delta_{2}$, as expected.

A comment on the values of the shape parameter chosen for the simulation study, namely $\alpha=$ $0.1,0.3,0.5,0.75$ and 1.0, is in order. Following Dupuis and Mills (1998), in most practical applications, $0<\alpha<1$; see, for instance, the applications considered in Sanhueza et al. (2008) and Leiva et al. (2008). As showed in Høyland and Rausand (1994, p. 46-48), this follows from the derivation of the $\mathcal{B S}$ distribution. As a result, most of the simulation experiments for the $\mathcal{B S}$ distribution have been conducted for values of $\alpha$ between 0 and 1; see, for example, Dupuis and Mills (1998), Wu and Wong (2004), Ng et al. (2006), Wang et al. (2006), Lemonte et al. (2007, 2008) and Cysneiros et al. (2008). Likewise, we chose values of $\alpha$ in the unit interval to run our simulations and to analyse the simulation findings in detail. We now briefly comment on simulation results for larger values of $\alpha$. We conducted Monte Carlo experiments for various values of $\alpha>1$. In general, for testing on $\alpha$ and $\beta$, the likelihood ratio test is oversized and the gradient test becomes conservative. However, the best performing test is the gradient test. For example, when $n=20$, d.o.c. $=10 \%$, $\gamma=10 \%$ and $\alpha=1.8$, the null rejection rates of the tests were $12.44 \%\left(L R_{(\alpha)}\right), 9.77 \%\left(S_{T(\alpha)}\right)$ and $9.83 \%\left(S_{T(\alpha)}^{*}\right)$ for testing $\mathcal{H}_{00}: \alpha=1.8$, and $11.90 \%\left(L R_{(\beta)}\right)$ and $9.09 \%\left(S_{T(\beta)}\right)$ for testing $\mathcal{H}_{01}: \beta=1$.

A natural question at this point is why the Wald and the Rao score tests were not included in the previous simulation experiments. Recall that the Wald and the score statistics involve the Fisher information matrix, which cannot be obtained for the $\mathcal{B S}$ distribution under type-II censoring. A common practice in such a case is to use the observed information in the place of the expected information. We followed this approach and ran various simulation experiments including the Wald and the score tests. Our general conclusion is that these tests cannot be recommended for the following reasons. First, the Wald and score tests were markedly oversized. For example, for $n=20, \gamma=10 \%$ and $\alpha=0.75$, the null rejection rates of the Wald and score tests for testing the null hypothesis $\mathcal{H}_{01}: \beta=1$ are, respectively, $13.79 \%$ and $15.61 \%$ for d.o.c. $=10 \%, 16.73 \%$ and $15.44 \%$ for d.o.c. $=30 \%$ and $19.02 \%$ and $13.70 \%$ for d.o.c. $=50 \%$. Second, in our simulations the inverse of the observed information matrix frequently produced negative standard errors in censored samples.

In summary, the best performing test for $\alpha$ is the adjusted gradient test, i.e. the one that uses the bias-corrected estimator of $\alpha$. As far as hypothesis testing on $\beta$ is concerned, the gradient test performs better than the likelihood ratio test and should be preferred. 
Table 3: Nonnull rejection rates (\%): $\alpha=0.5, \beta=1, \gamma=10 \%$ and different sample sizes.

\begin{tabular}{|c|c|c|c|c|c|c|c|c|}
\hline \multirow[b]{2}{*}{$n$} & \multirow{2}{*}{$\begin{array}{c}\text { d.o.c. } \\
(\%)\end{array}$} & \multicolumn{4}{|c|}{$\mathcal{H}_{10}: \alpha=\delta_{1}$} & \multicolumn{3}{|c|}{$\mathcal{H}_{11}: \beta=\delta_{2}$} \\
\hline & & $\delta_{1}$ & $L R_{(\alpha)}$ & $S_{T(\alpha)}$ & $S_{T(\alpha)}^{*}$ & $\delta_{2}$ & $L R_{(\beta)}$ & $\overline{S_{T(\beta)}}$ \\
\hline \multirow[t]{12}{*}{80} & 0 & 0.50 & 10.71 & 10.11 & 10.25 & 1.00 & 10.53 & 10.20 \\
\hline & & 0.54 & 23.90 & 25.84 & 27.97 & 1.04 & 19.06 & 19.50 \\
\hline & & 0.58 & 56.16 & 58.99 & 62.18 & 1.10 & 54.60 & 55.66 \\
\hline & & 0.60 & 71.59 & 73.97 & 76.45 & 1.13 & 73.29 & 73.96 \\
\hline & 20 & 0.50 & 10.65 & 9.55 & 9.68 & 1.00 & 10.55 & 10.10 \\
\hline & & 0.54 & 19.34 & 20.93 & 23.62 & 1.04 & 18.67 & 19.27 \\
\hline & & 0.58 & 44.89 & 48.51 & 52.02 & 1.10 & 52.70 & 54.15 \\
\hline & & 0.60 & 58.92 & 62.02 & 65.53 & 1.13 & 70.86 & 72.06 \\
\hline & 40 & 0.50 & 10.93 & 9.92 & 9.72 & 1.00 & 10.30 & 9.51 \\
\hline & & 0.54 & 15.74 & 17.20 & 19.83 & 1.04 & 17.34 & 18.47 \\
\hline & & 0.58 & 33.78 & 37.30 & 40.92 & 1.10 & 47.69 & 50.16 \\
\hline & & 0.60 & 44.65 & 48.93 & 52.86 & 1.13 & 64.54 & 67.06 \\
\hline \multirow[t]{12}{*}{120} & 0 & 0.50 & 10.53 & 10.17 & 10.18 & 1.00 & 10.62 & 10.37 \\
\hline & & 0.54 & 30.34 & 32.59 & 34.90 & 1.04 & 22.74 & 23.25 \\
\hline & & 0.58 & 72.73 & 74.76 & 76.52 & 1.10 & 69.69 & 70.35 \\
\hline & & 0.60 & 86.61 & 87.73 & 88.78 & 1.13 & 87.01 & 87.47 \\
\hline & 20 & 0.50 & 10.64 & 10.02 & 10.14 & 1.00 & 10.19 & 9.80 \\
\hline & & 0.54 & 24.58 & 26.43 & 28.95 & 1.04 & 22.78 & 23.46 \\
\hline & & 0.58 & 60.06 & 62.86 & 65.65 & 1.10 & 68.10 & 69.23 \\
\hline & & 0.60 & 75.07 & 77.04 & 79.19 & 1.13 & 84.79 & 85.37 \\
\hline & 40 & 0.50 & 10.65 & 9.90 & 9.81 & 1.00 & 10.21 & 9.65 \\
\hline & & 0.54 & 19.02 & 21.07 & 23.71 & 1.04 & 20.63 & 22.17 \\
\hline & & 0.58 & 45.24 & 48.49 & 52.05 & 1.10 & 60.84 & 62.71 \\
\hline & & 0.60 & 59.85 & 62.66 & 65.77 & 1.13 & 79.08 & 80.55 \\
\hline \multirow[t]{12}{*}{150} & 0 & 0.50 & 9.98 & 9.72 & 9.76 & 1.00 & 10.15 & 10.00 \\
\hline & & 0.54 & 35.53 & 38.04 & 40.24 & 1.04 & 26.34 & 26.97 \\
\hline & & 0.58 & 80.99 & 82.36 & 83.63 & 1.10 & 77.31 & 77.87 \\
\hline & & 0.60 & 92.42 & 93.24 & 93.82 & 1.13 & 92.02 & 92.33 \\
\hline & 20 & 0.50 & 10.13 & 9.94 & 9.99 & 1.00 & 10.39 & 10.15 \\
\hline & & 0.54 & 28.30 & 30.38 & 33.01 & 1.04 & 25.52 & 26.39 \\
\hline & & 0.58 & 68.24 & 70.56 & 73.17 & 1.10 & 75.83 & 76.92 \\
\hline & & 0.60 & 83.46 & 84.88 & 86.39 & 1.13 & 91.42 & 91.90 \\
\hline & 40 & 0.50 & 10.51 & 10.06 & 9.85 & 1.00 & 10.33 & 10.02 \\
\hline & & 0.54 & 21.85 & 23.99 & 26.53 & 1.04 & 22.49 & 23.86 \\
\hline & & 0.58 & 53.25 & 56.23 & 59.28 & 1.10 & 68.41 & 70.32 \\
\hline & & 0.60 & 68.45 & 70.74 & 73.44 & 1.13 & 86.73 & 87.78 \\
\hline
\end{tabular}




\section{Applications}

In this section, we shall illustrate the use of the likelihood ratio and gradient tests in three real data sets. The first data set, collected by members of the Instrument Development Unit of the Physical Research Staff, Boeing Airplane Company, are the number of cycles-to-failure of 101 strips of 6061T6 aluminum sheeting, cut parallel to the direction of rolling. Each had been subjected to periodic loading with a frequency of 18 cycles per second, and maximum stress of $31 \mathrm{kpsi}$. The data were taken from Table 8 of Lemonte et al. (2007). Suppose we are interested in testing $\mathcal{H}_{00}: \alpha=0.15$ against $\mathcal{H}_{10}: \alpha \neq 0.15$ and $\mathcal{H}_{01}: \beta=125$ against $\mathcal{H}_{11}: \beta \neq 125$. These hypotheses were considered in Cysneiros et al. (2008). Assuming different censoring proportions, the observed values of the different test statistics and the corresponding $p$-values are given in Table 4 . Note that for complete data without censoring $(m=101)$ and when $m=80$, at the 5\% significance level, the likelihood ratio test does not reject the null hypothesis $\mathcal{H}_{00}$ unlike the gradient tests. For the other values of $m$, the likelihood ratio and the gradient tests lead to the same conclusion. In all cases, the gradient tests provide stronger evidence against the null hypothesis (smaller $p$-values than the likelihood test). For testing the null hypothesis $\mathcal{H}_{01}$, the same decision is reached by the likelihood ratio and gradient tests, i.e. the null hypothesis $\mathcal{H}_{01}$ is rejected at any usual significance level. We also note that as the censoring proportions increase, the $p$-values decrease.

Table 4: Test statistics ( $p$-values between parentheses); aluminum fatigue data.

\begin{tabular}{|c|c|c|c|c|c|}
\hline \multirow[b]{2}{*}{$m$} & \multicolumn{3}{|c|}{ Inference on $\alpha$} & \multicolumn{2}{|c|}{ Inference on $\beta$} \\
\hline & $L R_{(\alpha)}$ & $S_{T(\alpha)}$ & $S_{T(\alpha)}^{*}$ & $L R_{(\beta)}$ & $S_{T(\beta)}$ \\
\hline 101 & $3.5771(0.0586)$ & $3.9841(0.0459)$ & $4.3171(0.0377)$ & $9.4279(0.0021)$ & $9.2402(0.0024)$ \\
\hline 95 & $2.8573(0.0910)$ & $3.1598(0.0755)$ & $3.4821(0.0620)$ & $9.4250(0.0021)$ & $9.2582(0.0023)$ \\
\hline 90 & $3.0826(0.0791)$ & $3.4342(0.0639)$ & $3.7969(0.0513)$ & $9.5167(0.0020)$ & $9.3800(0.0022)$ \\
\hline 80 & $3.8361(0.0502)$ & $4.3641(0.0367)$ & $4.8300(0.0280)$ & $9.8999(0.0017)$ & $9.8573(0.0017)$ \\
\hline 70 & $2.8684(0.0903)$ & $3.2360(0.0720)$ & $3.6615(0.0557)$ & $9.4412(0.0021)$ & $9.5939(0.0020)$ \\
\hline 60 & $4.5172(0.0336)$ & $5.3218(0.0211)$ & $5.9240(0.0149)$ & $10.8407(0.0010)$ & $11.3070(0.0008)$ \\
\hline 50 & $4.0608(0.0439)$ & $4.8212(0.0281)$ & $5.4073(0.0201)$ & $10.3798(0.0013)$ & $11.3522(0.0008)$ \\
\hline 40 & $8.8234(0.0030)$ & $11.6943(0.0006)$ & $12.7236(0.0004)$ & $15.3808(0.0001)$ & $18.0787(0.0000)$ \\
\hline
\end{tabular}

As a second application, we consider the data provided by $\mathrm{McCool}$ (1974) on the the lifetime, in hours, of 10 sustainers of a certain type. The data, used by Cohen et al. (1984) as an illustration of the three-parameter Weibull distribution, can be found at Table 5 of Lemonte et al. (2007). We wish to test $\mathcal{H}_{00}: \alpha=0.21$ against $\mathcal{H}_{10}: \alpha \neq 0.21$ and $\mathcal{H}_{01}: \beta=180$ against $\mathcal{H}_{11}: \beta \neq 180$. These hypotheses were considered in Cysneiros et al. (2008). Under different censoring proportions, the observed values of the different test statistics and the corresponding $p$-values are given in Table 5 . Note that for complete data (without censoring) the likelihood ratio test does not reject the null hypothesis $\mathcal{H}_{00}$ at any usual significance level, whereas the original and adjusted gradient tests reject the null hypothesis $\mathcal{H}_{00}$ at the $10 \%$ and $5 \%$ significance levels, respectively. Except for the case where 
$m=10$ (no censoring), the likelihood and gradient tests lead to the same conclusion. For testing the null hypothesis $\mathcal{H}_{01}$, unless $m=7$, the same decision is reached by the likelihood ratio and gradient tests, i.e. the null hypothesis is rejected at the $10 \%$ significance level for $m=8,9$ and 10 , and it not rejected at any usual significance level for $m=6$.

Table 5: Test statistics ( $p$-values between parentheses); sustainers data.

\begin{tabular}{ccccccc}
\hline & \multicolumn{3}{c}{ Inference on $\alpha$} & & \multicolumn{2}{c}{ Inference on $\beta$} \\
\cline { 2 - 4 } \cline { 6 - 7 } \cline { 6 - 7 } & $L R_{(\alpha)}$ & $S_{T(\alpha)}$ & $S_{T(\alpha)}^{*}$ & & $L R_{(\beta)}$ & $S_{T(\beta)}$ \\
\hline 10 & $2.1646(0.1412)$ & $2.7944(0.0946)$ & $4.0043(0.0454)$ & & $2.9417(0.0863)$ & $2.7580(0.0968)$ \\
9 & $0.0770(0.7814)$ & $0.0728(0.7873)$ & $0.0000(1.0000)$ & & $3.2449(0.0716)$ & $2.9248(0.0872)$ \\
8 & $0.3307(0.5653)$ & $0.2911(0.5895)$ & $0.0000(1.0000)$ & & $3.1616(0.0754)$ & $2.8499(0.0914)$ \\
7 & $0.6732(0.4119)$ & $0.5514(0.4578)$ & $0.1472(0.7013)$ & & $2.9510(0.0858)$ & $2.7036(0.1001)$ \\
6 & $0.8471(0.3574)$ & $0.6620(0.4159)$ & $0.2234(0.6365)$ & & $2.6797(0.1016)$ & $2.5463(0.1106)$ \\
\hline
\end{tabular}

Finally, our third example uses the data given by Gupta (1952), which are the survival times (in days) of the first seven of a sample of ten mice after being inoculated with a culture of tuberculosis. The observations are: $41,44,46,54,55,58$ and 60. Suppose we are interested in testing $\mathcal{H}_{00}: \alpha=0.1$ against $\mathcal{H}_{10}: \alpha \neq 0.1$ and $\mathcal{H}_{01}: \beta=54$ against $\mathcal{H}_{11}: \beta \neq 54$. We have $L R_{(\alpha)}=1.7607$ ( $p$-value $=0.1845), S_{T(\alpha)}=2.3152(p$-value $=0.1281), S_{T(\alpha)}^{*}=3.7346(p$-value $=0.0533), L R_{(\beta)}=1.3710$ $(p$-value $=0.2416)$ and $S_{T(\beta)}=1.2054(p$-value $=0.2722)$. Note that the likelihood ratio and original gradient tests do not reject the null hypothesis $\mathcal{H}_{00}$ at any usual significance level, whereas the adjusted gradient test rejects the null hypothesis $\mathcal{H}_{00}$ at the $10 \%$ level. Additionally, the null hypothesis $\mathcal{H}_{01}$ is not rejected at any usual significance level based on the statistics $L R_{(\beta)}$ and $S_{T(\beta)}$.

\section{Concluding remarks}

The $\mathcal{B S}$ distribution is becoming increasingly popular in lifetime analyses and reliability studies. In this paper, we dealt with the issue of performing hypothesis testing concerning the parameters of this distribution under type-II right censored samples. We considered the likelihood ratio and a recently proposed test, the gradient test. As evidenced by our simulation results, in general the likelihood ratio test is oversized (liberal), whereas the gradient test is undersized (conservative) and less size distorted than the likelihood ratio test. We also considered an adjusted gradient statistic for testing hypotheses on the parameter $\alpha$ whose null distribution is more accurately approximated by the limiting null distribution than that of the original gradient test statistic. Overall, our numerical results favor the gradient tests for testing hypotheses on the parameters $\alpha$ and $\beta$. The results in this paper are encouraging and motivate further work. We plan to investigate the performance of the gradient test as compared to that of the likelihood ratio test in the Birnbaum-Saunders regression model under various censoring mechanisms. 


\section{References}

Aslam, M., Jun, C.H., Ahmad, M. (2010). New acceptance sampling plans based on life tests for Birnbaum-Saunders distributions. Journal of Statistical Computation and Simulation. DOI : $10.1080 / 00949650903418883$.

Balakrishnan, N., Cohen, A.C. (1991). Order Statistics and Inference: Estimation Methods. Academic Press: San Diego.

Balakrishnan, N., Leiva, V., López, J. (2007). Acceptance sampling plans from truncated life tests from generalized Birnbaum-Saunders distribution. Communications in Statistics - Simulation and Computation 36, 643-656.

Bhattacharyya, G.K., Fries, A. (1982). Fatigue failure models: Birnbaum-Saunders versus inverse Gaussian. IEEE Transactions on Reliability 31, 439-440.

Bhatti, C.R. (2010). The Birnbaum-Saunders autoregressive conditional duration model. Mathematics and Computers in Simulation 80, 2062-2078.

Birnbaum, Z.W., Saunders, S.C. (1969a). A new family of life distributions. Journal of Applied Probability 6, 319-327.

Birnbaum, Z.W., Saunders, S.C. (1969b). Estimation for a family of life distributions with applications to fatigue. Journal of Applied Probability 6, 328-377.

Castillo, N.O., Gómez, H.W., Bolfarine, H. (2009). Epsilon Birnbaum-Saunders distribution family: properties and inference. Statistical Papers. DOI :10.1007/s00362-009-0293-x.

Cohen, A.C., Whitten, B.J., Ding, Y. (1984). Modified moment estimation for the three-parameter Weibull distribution. Journal of Quality Technology 16, 159-167.

Cysneiros, A.H.M.A, Cribari-Neto, F., Araújo-Jr, C.A.G. (2008). On Birnbaum-Saunders inference. Computational Statistics ans Data Analysis 52, 4939-4950.

Desmond, A.F. (1985). Stochastic models of failure in random environments. Canadian Journal of Statistics 13, 171-183.

Desmond, A.F. (1986). On the relationship between two fatigue-life models. IEEE Transactions on Reliability 35, 167-169.

Díaz-García, J.A., Leiva, V. (2005). A new family of life distributions based on the elliptically contoured distributions. Journal of Statistical Planning and Inference 128, 445-457.

Doornik, J.A. (2006). An Object-Oriented Matrix Language - Ox 4, 5th ed. Timberlake Consultants Press: London. 
Dupuis, D.J., Mills, J.E. (1998). Robust estimation of the Birnbaum-Saunders distribution. IEEE Transactions on Reliability 47, 88-95.

From, S.G., Li, L. (2006). Estimation of the parameters of the Birnbaum-Saunders distribution. Communications in Statistics - Theory and Methods 35, 2157-2169.

Gómes, H.W., Olivares-Pacheco, J.F., Bolfarine, H. (2009). An extension of the generalized BirnbaumSaunders distribution. Statistics and Probability Letters 79, 331-338.

Guiraud, P., Leiva, V., Fierro, R. (2009). A non-central version of the Birnbaum-Saunders distribution for reliability analysis. IEEE Transactions on Reliability 58, 152-160.

Gupta, A.K. (1952). Estimation of the mean and standard deviation of a normal population from a censored sample. Biometrika 39, 260-273

Høyland, A., Rausand, M. (1994). System Reliability Theory - Models and Statistical Methods. John Wiley and Sons: New York.

Jeng, S.L. (2003). Inferences for the fatigue life model based on the Birnbaum-Saunders distribution. Communications in Statistics - Simulation and Computation 32, 43-60.

Kundu, D., Kannan, N., Balakrishnan, N. (2008). On the function of Birnbaum-Saunders distribution and associated inference. Computational Statistics and Data Analysis 52, 2692-2702.

Leiva, V., Barros, M., Paula, G.A., Sanhueza, A. (2008). Generalized Birnbaum-Saunders distributions applied to air pollutant concentration. Environmetrics 19, 235-249 .

Leiva, V., Sanhueza, A., Angulo, J.M. (2009). A length-biased version of the Birnbaum-Saunders distribution with application in water quality. Stochastic Environmental Research and Risk Assessment 23, 299-307.

Lemonte, A.J., Cribari-Neto, F., Vasconcellos, K.L.P. (2007). Improved statistical inference for the twoparameter Birnbaum-Saunders distribution. Computational Statistics and Data Analysis 51, 4656-4681.

Lemonte, A.J., Ferrari, S.L.P. (2010a). The local power of the gradient test. Annals of the Institute of Statistical Mathematics. DOI:10.1007/s10463-010-0315-4.

Lemonte, A.J., Ferrari, S.L.P. (2010b). Size and power properties of some tests in the Birnbaum-Saunders regression model. Computational Statistics and Data Analysis. To appear.

Lemonte, A.J., Simas, A.B., Cribari-Neto, F. (2008). Bootstrap-based improved estimators for the twoparameter Birnbaum-Saunders distribution. Journal of Statistical Computation and Simulation 78, 37-49.

McCool, J.I. (1974). Inferential techniques for Weibull populations. Aerospace Research Laboratories Report ARL TR74-0180. Wright-Patterson Air Force Base, Dayton, OH.

Ng, H.K.T., Kundu, D., Balakrishnan, N. (2006). Point and interval estimation for the two-parameter Birnbaum-Saunders distribution based on Type-II censored samples. Computational Statistics ans Data Analysis 50, 3222-3242. 
Nocedal, J., Wright, S.J. (1999). Numerical Optimization. Springer: New York.

Press, W.H., Teulosky, S.A., Vetterling, W.T., Flannery, B.P. (2007). Numerical Recipes in C: The Art of Scientific Computing, 3rd ed. Cambridge University Press.

Rieck, J.R. (1995). Parameter estimation for the Birnbaum-Saunders distribution based on symmetrically censored samples. Communications in Statistics - Theory and Methods 24, 1721-1736.

Rieck, J.R. (1999). A moment-generating function with application to the Birnbaum-Saunders distribution. Communications in Statistics - Theory and Methods 28, 2213-2222.

Sanhueza, A., Leiva, V., Balakrishnan, N. (2008). The generalized Birnbaum-Saunders distribution and its theory, methodology, and application. Communications in Statistics - Theory and Methods 37, 645-670.

Saunders, S.C. (1974). A family of random variables closed under reciprocation. Journal of the American Statistical Association 69, 533-539.

Terrell, G.R. (2002). The gradient statistic. Computing Science and Statistics 34, 206-215.

Xu, A., Tang, Y. (2010). Reference analysis for Birnbaum-Saunders distribution. Computational Statistics and Data Analysis 54, 185-192.

Wang, Z., Desmond, A.F., Lu, X. (2006). Modified censored moment estimation for the two-parameter Birnbaum-Saunders distribution. Computational Statistics ans Data Analysis 50, 1033-1051.

Wu, J., Wong, A.C.M. (2004). Improved interval estimation for the two-parameter Birnbaum-Saunders distribution. Computational Statistics and Data Analysis 47, 809-821. 